\title{
Fatores associados à subestimação materna do peso da criança: um estudo de base populacional
}

\author{
Factors associated with maternal \\ underestimation of child's weight: A \\ population-based study
}

Thais Parreira de Deus Araújo FREITAS

Lara Livia Santos da SILVA²

Gabriela Silva TELES ${ }^{1}$

Maria do Rosário Gondim PEIXOTO²

Ida Helena Carvalho Francescantonio MENEZES²

\section{R E S U M O}

\section{Objetivo}

Investigar os fatores associados à subestimação materna do peso de crianças menores de cinco anos de Goiânia, Goiás.

\section{Métodos}

Estudo transversal, de base populacional, domiciliar, com 673 crianças menores de cinco anos de idade. A percepção materna do peso da criança foi obtida pela pergunta: "Atualmente, o que a senhora (mãe) acha do peso do seu filho?", e o estado nutricional da criança foi avaliado pelo índice de massa corporal por idade. Regressão múltipla de Poisson foi realizada a um nível de significância de 5\%. Para a análise de concordância, foi utilizado o teste Kappa.

\section{Resultados}

Das crianças estudadas, 1,6\% estavam magras, $87,7 \%$ eutróficas e 10,7\% com excesso de peso. A maioria das mães $(66,0 \%)$ identificou corretamente o peso de seus filhos; $5,2 \%$ superestimaram, e $28,8 \%$ subestimaram. Mães de crianças com excesso de peso, idade superior a 24 meses, apresentaram maior prevalência de subestimaçãa, enquanto as mães com excesso de peso e maior escolaridade apresentaram menor subestimação do

\footnotetext{
1 Universidade Federal de Goiás, Faculdade de Nutrição, Curso de Nutrição. Goiânia, GO, Brasil.

2 Universidade Federal de Goiás, Faculdade de Nutrição, Programa de Pós-Graduação em Nutrição e Saúde. R. 227, Qd. 68, s/n., Setor Leste Universitário, 74605-080, Goiânia, Goiás, Brasil. Correspondência para/Correspondence to: IHCF MENEZES. E-mail: <idahelenamenezes@gmail.com>.

Apoio: Fundação de Amparo à Pesquisa do Estado de Goiás (Processo nº 201010267000924).
} 
peso das crianças. A concordância entre a opinião materna sobre o peso da criança e o real estado nutricional da criança foi de $0,12(p<0,001)$.

\section{Conclusão}

Existe uma distorção na percepção materna do peso de crianças menores de cinco anos. A subestimação materna do peso da criança apresentou associação direta com idade e estado nutricional da criança e associação inversa com escolaridade e estado nutricional materno. Sugere-se atenção no atendimento a essas mães com o objetivo de evitar o ganho de peso excessivo nessa idade e prejuízos na saúde da criança.

Palavras-chave: Estado nutricional. Lactente. Mães. Percepção de peso. Pré-escolar.

\section{A B S T R A C T}

\section{Objective}

To investigate the factors associated with maternal underestimation of weight status of children under five years in Goiânia, Goiás, Brazil.

\section{Methods}

A population-based cross-sectional study involving 673 children under five years of age was carried out. Maternal perception of child's weight was assessed by the question: "What do you think about your child's current weight?". The child's nutritional status was determined in terms of body mass index-for age. Poisson regression was used at 5\% significance level. Concordance analysis was performed using the Kappa test.

\section{Results}

Among the children studied, $1.6 \%$ were lean, $87.7 \%$ eutrophic, and $10.7 \%$ overweight. Most mothers (66.0\%) correctly classified the weight of their children, $5.2 \%$ overestimated it, and $28.8 \%$ underestimated it. Mothers of overweight children and those whose children were older than 24 months had a higher prevalence of underestimation of weight, whereas the overweight mothers and those with higher education level had a lower prevalence of underestimation of weight of their children. The correlation between maternal perception of the chil's weight and the actual child's nutritional status was $0.12(p<0.001)$.

\section{Conclusion}

Maternal misperception of weight of children under five years was observed. Maternal underestimation of their child's weight was directly associated with age and nutritional status of the child, and it was inversely associated with maternal education level and nutritional status. More attention should be paid to these mothers in order to prevent excessive weight gain at this age and further harm to children's health.

Keywords: Nutritional status. Infant. Mother. Weight perception. Child, preschool.

\section{N T R O D U Ç Ã O}

A Organização Mundial da Saúde (OMS) estima que nos próximos anos o excesso de peso atinja aproximadamente 42 milhões de crianças menores de cinco anos de idade, sendo a maioria residente em países em desenvolvimento'.

Crianças que desenvolvem obesidade têm maior probabilidade de permanecerem obesas na fase adulta e de desenvolverem morbidades mais graves e mortalidade precoce do que adultos cuja obesidade teve início mais tardiamente ${ }^{2-4}$. Nessa fase, as decisões sobre disponibilidade de alimentos e as oportunidades para atividade física são principalmente controladas pelos pais e outros cuidadores.

Assim, destaca-se a importância do vínculo mãe e filho na recuperação e no tratamento da condição de saúde, uma vez que a figura materna está geralmente mais envolvida no processo do cuidado familiar. A percepção da mãe sobre o estado nutricional do filho será determinante na escolha do padrão de cuidados que irão definir tanto a alimentação fora de casa quanto os hábitos alimentares diários, que afetam diretamente o estado nutricional da criança ${ }^{5-7}$.

Pais com dificuldades em reconhecer adequadamente o estado nutricional de seus filhos 
agravam o problema por falta de intervenção precoce. Tal dificuldade tem sido citada como uma das razões que contribuem para o aumento do excesso de peso entre as crianças ${ }^{7}$. Vários fatores são descritos como limitantes para a percepção adequada, tais como: escolaridade e idade materna, trabalho da mãe fora do domicílio, classe socioeconômica, sexo e idade da criança ${ }^{8-11}$.

Apesar da relevância do conhecimento da percepção materna do estado nutricional do seu filho e dos múltiplos fatores que podem condicionar essa percepção, poucos estudos trataram do tema. Em recente revisão sistemática sobre a percepção das mães quanto ao estado nutricional de seus filhos, foram identificados 17 estudos, sendo apenas 5 com amostra de crianças menores de cinco anos ${ }^{12}$. Acrescenta-se que, no Brasil, os estudos que abordaram essa temática entre crianças menores de cinco anos ${ }^{9,13}$ não eram de base populacional, o que dificulta a generalização dos resultados.

Nesse sentido, o presente estudo objetivou investigar os fatores associados à subestimação materna do peso de crianças menores de cinco anos de uma capital do Brasil.

\section{M É T O D O S}

O presente trabalho faz parte de um estudo epidemiológico, de base populacional, denominado "Perfil nutricional de crianças menores de cinco anos". Trata-se de estudo transversal, com amostra constituída de crianças menores de cinco anos, de ambos os sexos, residentes na zona urbana da cidade de Goiânia, Goiás, Brasil.

O cálculo do tamanho amostral foi realizado para o estudo matriz, considerando 10,0\% de excesso de peso, um nível de significância de 0,05 , erro absoluto de $2,5 \%$ e efeito do delineamento (conglomerados) de 1,5\%, o que resultou em uma amostra desejável mínima de 829 crianças. O processo de amostragem foi em múltiplos estágios; no primeiro, foi realizado um sorteio aleatório de 87 setores censitários da cidade de Goiânia (GO) ${ }^{14}$; no segundo, o sorteio do domicílio participante; o terceiro estágio correspondeu ao sorteio da criança, caso houvesse mais de uma criança menor de cinco anos de idade na residência. Obteve-se um total de 832 crianças nessa faixa etária; após a exclusão das crianças gêmeas $(n=12)$, sem a medida de peso ou estatura $(n=35)$ e sem a percepção materna sobre o peso $(n=112)$, a amostra analisada ficou constituída por 673 crianças. Cálculo a posteriori demonstrou que essa amostra permitiu detectar a subestimação do peso da criança, com um erro absoluto de 2,6\%, considerando prevalência de subestimação do peso em crianças de $38,8 \%{ }^{5}$.

A coleta de dados foi feita entre setembro de 2011 e outubro de 2012. O instrumento utilizado foi um questionário padronizado, respondido pela mãe da criança e aplicado por dupla de entrevistadores habilitados.

A percepção da mãe sobre o peso de seu filho foi obtida por meio da pergunta: "Atualmente, o que a senhora (mãe) acha do peso do seu filho?", e as respostas foram classificadas em baixo, adequado e excessivo. A partir dessas respostas, foi definida a variável subestimação materna do peso da criança (não/sim), da seguinte maneira: Não - quando a mãe percebia que o peso da criança estava de acordo com o real estado nutricional ou em categoria superior a este; Sim - quando a mãe percebia que o peso da criança estava em uma categoria inferior ao seu real estado nutricional. Considerou-se a variável subestimação materna do peso da criança como a variável dependente nas análises.

As variáveis independentes relacionadas às crianças foram: sexo (masculino/feminino); idade em anos completos ( $<24$ meses $/ \geq 24$ meses); prematuridade (sim/não); primogênito (sim/ não); peso ao nascer (baixo peso ao nascer/peso adequado); e estado nutricional classificado pelo Índice de Massa Corporal por Idade (IMC/I), obtido pela equação peso/altura². Para a classificação do baixo peso ao nascer, considerou-se como ponto de corte o percentil 10 das curvas de peso ao 
nascer específicas para crianças nascidas a termo ${ }^{15}$ e pré-termo ${ }^{16}$ recentemente publicadas.

As medidas do peso e da estatura das crianças foram realizadas utilizando procedimentos padronizados ${ }^{17}$. O peso foi coletado por meio de balança digital G-Tech@, com capacidade de $150 \mathrm{~kg}$ e precisão de $100 \mathrm{~g}$, com a criança vestida com o mínimo de roupa possível. A estatura das crianças menores de dois anos foi verificada com o auxílio de uma régua antropométrica de madeira, com comprimento de $100 \mathrm{~cm}$ e precisão de $0,1 \mathrm{~cm}$. Já a estatura das crianças com idade superior a dois anos foi aferida utilizando-se fita métrica inelástica, com precisão de $0,1 \mathrm{~cm}$, fixada à parede, sem rodapé, sendo a medida realizada com auxílio de um esquadro de madeira. Para a avaliação do estado nutricional, utilizou-se o software World Health Organization Anthro versão 3.2.2. O IMC/I foi classificado de acordo com os valores de escore Z em: <-2 (magreza); $\geq-2$ e $\leq+2$ (eutrofia + risco de sobrepeso); $>+2$ (excesso de peso) ${ }^{18}$.

As variáveis independentes relacionadas às mães foram: idade ( $<20$ anos e $\geq 20$ anos); escolaridade (ensino fundamental, médio e superior); e estado nutricional classificado pelo IMC, segundo a $\mathrm{OMS}^{19,20}$ a partir do peso e da estatura referidos ${ }^{21}$.

Os dados foram digitados no programa Epi Info versão 3.5.2, e a análise estatística foi realizada no Stata 12.0. As associações bivariadas entre as variáveis categóricas e o estado nutricional da criança foram analisadas pelo teste exato de Fisher. Para a análise de concordância entre estado nutricional e percepção materna do peso da criança foi utilizado o teste Kappa, e sua interpretação ocorreu considerando valores de Kappa $<0,20$ (concordância fraca), $\geq 0,20 a<0,40$ (concordância regular), $\geq 0,40 a<0,60$ (concordância moderada), $\geq 0,60$ a <0,80 (concordância boa) e $\geq 0,80$ (concordância quase perfeita) ${ }^{22}$. Regressão múltipla de Poisson com estimativa robusta da variância foi utilizada para identificar preditores da subestimação materna do peso da criança (variável dependente). Foram testadas no modelo ajustado as variáveis que na análise de regressão bruta apresentaram significância menor que 20\% $(p<0,20)$. A magnitude da associação entre as variáveis independentes e a ocorrência da variável desfecho foi expressa em Razão de Prevalência (RP) e respectivos Intervalos de Confianças de 95\% (IC95\%), considerando-se significativo $p<0,05$.

O projeto foi aprovado pelo Comitê de Ética em Pesquisa da Universidade Federal de Goiás (Protocolo n 074/11). Os responsáveis autorizaram a participação na pesquisa a partir da assinatura do Termo de Consentimento Livre Esclarecido.

\section{RESULTADOS}

Do total de 673 crianças, 50,4\% eram do sexo masculino e 55,7\% eram maiores de 24 meses. Havia 9,4\% de crianças prematuras, 43,8\% primogênitas e 2,8\% nasceram com baixo peso. Quanto aos dados maternos, 92,3\% das mães tinham idade igual ou superior a 20 anos, 54,7\% tinham o ensino médio e 38,4\% delas estavam com excesso de peso. Em relação ao estado nutricional das crianças, 1,6\% estavam magras, $87,7 \%$ eutróficas ou em risco de sobrepeso e 10,7\% já apresentavam excesso de peso (Tabela 1).

Segundo a percepção materna, $23,0 \%$ das crianças estavam com baixo peso, $68,7 \%$ estavam com peso adequado e 8,3\% com excesso de peso. Considerando-se o peso real e a percepção materna do peso da criança, observou-se que as mães tendem a superestimar o peso das crianças magras e subestimar o peso das crianças com excesso de peso $(p<0,001)$, sendo que para as crianças que estavam abaixo do peso (magreza), $54,5 \%$ das mães perceberam o peso como sendo normal e 9,1\% como excessivo. Entre as crianças eutróficas/risco de sobrepeso, 25,2\% das mães perceberam o peso abaixo do normal e $4,8 \%$ consideraram o peso excessivo. Finalmente, entre as que estavam com excesso de peso, 59,7\% das mães consideraram o peso como adequado e 2,8\% como baixo peso (Tabela 2). Na análise para 
Tabela 1. Distribuição da amostra e associação dos dados da criança e maternos com o estado nutricional da criança (n=673). Goiânia (GO), 2012

\begin{tabular}{|c|c|c|c|c|c|}
\hline \multirow[b]{2}{*}{ Variáveis } & Total $n=673$ & \multicolumn{3}{|c|}{ Estado nutricional da criança } & \multirow[b]{2}{*}{$p^{* *}$} \\
\hline & $\%$ & $\begin{array}{c}\text { Magreza } \\
n=11 \quad(1,6 \%)\end{array}$ & $\begin{array}{c}\text { Eutrofia/Risco* } \\
n=590(87,7 \%)\end{array}$ & $\begin{array}{c}\text { Excesso de peso } \\
n=72 \quad(10,7 \%)\end{array}$ & \\
\hline \multicolumn{6}{|l|}{ Dados da criança } \\
\hline Sexo & & & & & 0,245 \\
\hline Feminino & $334(49,6)$ & $3(0,9)$ & $298(89,2)$ & $33(9,9)$ & \\
\hline Masculino & $339(50,4)$ & $8(2,4)$ & $292(86,1)$ & $39(11,5)$ & \\
\hline Idade & & & & & 0,042 \\
\hline$<24$ meses & $298(44,3)$ & $9(3,0)$ & $257(86,3)$ & $32(10,7)$ & \\
\hline$\geq 24$ meses & $375(55,7)$ & $2(0,5)$ & $333(88,8)$ & $40(10,7)$ & \\
\hline Prematuridade & & & & & 0,705 \\
\hline $\operatorname{sim}$ & $63(9,4)$ & $1(1,6)$ & $54(85,7)$ & $8(12,7)$ & \\
\hline Não & $610(90,6)$ & $10(1,6)$ & $536(87,9)$ & $64(10,5)$ & \\
\hline Primogênito & & & & & 0,510 \\
\hline Sim & $295(43,8)$ & $5(1,7)$ & $263(89,2)$ & $27(9,1)$ & \\
\hline Não & $378(56,2)$ & $6(1,6)$ & $327(86,5)$ & $45(11,9)$ & \\
\hline Peso ao nascer ${ }^{\mathrm{a}}$ & & & & & 0,172 \\
\hline Baixo peso ao nascer & $19(2,8)$ & $1(5,3)$ & $15(78,9)$ & $3(15,8)$ & \\
\hline Peso adequado & $647(97,2)$ & $10(1,6)$ & $569(87,9)$ & $68(10,5)$ & \\
\hline \multicolumn{6}{|l|}{ Dados maternos } \\
\hline Idade & & & & & 0,301 \\
\hline$<20$ anos & $52(7,7)$ & $2(3,9)$ & $44(84,6)$ & $6(11,5)$ & \\
\hline$\geq 20$ anos & $620(92,3)$ & $9(1,4)$ & $545(87,9)$ & $66(10,7)$ & \\
\hline Escolaridade & & & & & 0,008 \\
\hline Ensino fundamental & $165(24,5)$ & $2(1,2)$ & $151(91,5)$ & $12(7,3)$ & \\
\hline Ensino médio & $368(54,7)$ & $5(1,4)$ & $329(89,4)$ & $34(9,2)$ & \\
\hline Ensino superior & $140(20,8)$ & $4(2,8)$ & $110(78,6)$ & $26(18,6)$ & \\
\hline Estado nutricional'b & & & & & 0,439 \\
\hline Baixo peso & $46(7,4)$ & $2(4,3)$ & $39(84,8)$ & $5(10,9)$ & \\
\hline Eutrófica & $339(54,2)$ & $4(1,2)$ & $300(88,5)$ & $35(10,3)$ & \\
\hline Excesso de peso & $240(38,4)$ & $4(1,7)$ & $206(85,8)$ & $30(12,5)$ & \\
\hline
\end{tabular}

Nota: ${ }^{a} \mathrm{n}=666 ;{ }^{\mathbf{b}} \mathrm{n}=625 .{ }^{*}$ Risco de sobrepeso; ${ }^{* *}$ Teste exato de Fisher.

o total da amostra, a maioria das mães ( $n=444$; $66,0 \%$ ) teve uma percepção correta do peso da criança, 5,2\% ( $n=35)$ delas superestimaram e $28,8 \%(n=194)$ subestimaram o peso de seus filhos.

A concordância entre a percepção materna sobre o peso da criança e seu real estado nutricional, medida pelo teste Kappa, foi de 0,12 $(p<0,001)$, o que representa uma fraca concordância entre essas variáveis.

A Tabela 3 apresenta a prevalência de subestimação materna do peso da criança e a razão de prevalência bruta segundo as variáveis estudadas. Pode-se observar maior frequência de subestimação materna do peso para as crianças com idade igual ou superior a 24 meses $(33,1 \%)$ em relação às crianças menores de 24 meses $(23,5 \%)(p=0,006)$. Também foi observada maior subestimação do peso entre as crianças com excesso de peso $(62,5 \%)$ em relação àquelas sem excesso de peso $(24,8 \%)(p<0,01)$. As variáveis estudadas apresentaram $p<0,20$ na análise de regressão bruta, com exceção do sexo da criança e do peso ao nascer. 
Tabela 2. Associação entre estado nutricional da criança e a percepção materna do peso da criança. Goiânia (GO), 2012.

\begin{tabular}{|c|c|c|c|c|c|c|c|c|c|}
\hline \multirow{3}{*}{ Percepção materna } & & & \multicolumn{6}{|c|}{ Estado nutricional da criança } & \multirow{3}{*}{$p^{* *}$} \\
\hline & \multicolumn{2}{|c|}{ Total $(n=673)$} & \multicolumn{2}{|c|}{ Magreza $(n=11)$} & \multicolumn{2}{|c|}{ Eutrofia/Risco* $(n=590)$} & \multicolumn{2}{|c|}{ Excesso de peso $(n=72)$} & \\
\hline & $n$ & $\%$ & $n$ & $\%$ & $n$ & $\%$ & $n$ & $\%$ & \\
\hline & & & & & & & & & $<0,001$ \\
\hline Baixo & 155 & 23,0 & 4 & 36,4 & 149 & 25,2 & 2 & 2,8 & \\
\hline Adequado & 462 & 68,7 & 6 & 54,5 & 413 & 70,0 & 43 & 59,7 & \\
\hline Excessivo & 56 & 8,3 & 1 & 9,1 & 28 & 4,8 & 27 & 37,5 & \\
\hline
\end{tabular}

Nota: "Risco de sobrepeso; **Teste exato de Fisher.

Tabela 3. Prevalência de subestimação e Razão de Prevalência (RP) bruta e Intervalo de Confiança de 95\% (IC95\%) da subestimação materna do peso da criança segundo variáveis infantis e maternas (n=673). Goiânia (GO), 2012.

\begin{tabular}{|c|c|c|c|c|}
\hline Variáveis & Prevalência (IC95\%) & $p$ & RP bruta (IC95\%) & $p$ \\
\hline Sexo da criança & & 0,643 & & \\
\hline Feminino & $29,6(24,8 ; 34,8)$ & & 1,00 & \\
\hline Masculino & $28,0(23,3 ; 33,1)$ & & $0,94(0,74-1,20)$ & 0,644 \\
\hline Idade da criança & & 0,006 & & \\
\hline$<24$ meses & $23,5(18,8 ; 28,7)$ & & 1,00 & \\
\hline$\geq 24$ meses & $33,1(28,3 ; 38,1)$ & & $1,41(1,10-1,81)$ & 0,007 \\
\hline Prematuridade & & 0,088 & & \\
\hline Sim & $38,1(26,1 ; 51,2)$ & & 1,00 & \\
\hline Não & $27,9(24,3 ; 31,6)$ & & $0,73(0,52-1,03)$ & 0,072 \\
\hline Primogênito & & 0,085 & & \\
\hline Sim & $25,4(20,5 ; 30,8)$ & & 1,00 & \\
\hline Não & $31,5(26,8 ; 36,4)$ & & $1,24(0,97-1,58)$ & 0,088 \\
\hline Peso ao nascer & & 0,777 & & \\
\hline Baixo peso ao nascer & $31,6(12,6 ; 56,5)$ & & 1,00 & \\
\hline Peso adequado & $28,6(25,1 ; 32,2)$ & & $0,90(0,46-1,77)$ & 0,773 \\
\hline Estado nutricional da criança & & $<0,001$ & & \\
\hline Sem excesso de peso & $24,8(21,4 ; 28,4)$ & & 1,00 & \\
\hline Com excesso de peso & $62,5(50,3 ; 73,6)$ & & $2,52(2,01-3,16)$ & $<0,001$ \\
\hline Idade da mãe & & 0,110 & & \\
\hline$<20$ anos & $19,2(9,6 ; 32,5)$ & & 1,00 & \\
\hline$\geq 20$ anos & $29,7(26,1 ; 33,4)$ & & $1,54(0,87-2,73)$ & 0,136 \\
\hline Escolaridade da mãe & & 0,318 & & \\
\hline Ensino fundamental & $32,1(25,1 ; 39,8)$ & & 1,00 & \\
\hline Ensino médio & $29,1(24,5 ; 34,0)$ & & $0,90(0,69-1,19)$ & 0,475 \\
\hline Ensino superior & $24,3(17,4 ; 32,2)$ & & $0,76(0,52-1,09)$ & 0,136 \\
\hline Estado nutricional da mãe & & 0,409 & & \\
\hline Baixo peso & $37,0(23,2 ; 52,4)$ & & 1,00 & \\
\hline Eutrófica & $30,4(25,5 ; 35,6)$ & & $0,82(0,54-1,24)$ & 0,350 \\
\hline Excesso de peso & $27,5(21,9 ; 33,6)$ & & $0,74(0,48-1,14)$ & 0,178 \\
\hline
\end{tabular}

Na análise de regressão ajustada (Tabela 4), a prevalência de subestimação materna do peso das crianças com idade igual a 24 meses ou maior foi $29 \%$ maior do que entre as crianças com idade menor do que 24 meses $(R P=1,29$; $p=0,035)$. Já entre as crianças com excesso de 
peso, a prevalência de subestimação foi quase três vezes maior do que a observada entre as crianças sem excesso de peso ( $R P=2,70 ; p<0,001)$. Quanto às características maternas, permaneceram no modelo final a escolaridade e o estado nutricional da mãe, sendo que as mães com maior escolaridade ( $R P=0,63 ; p=0,017$ ) e com excesso de peso ( $R P=0,64 ; p=0,048$ ) apresentaram menores prevalências de subestimação do que as mães com ensino fundamental e baixo peso respectivamente.

Na Tabela 5, encontra-se a comparação dos nossos resultados com outros estudos sobre a subestimação materna do estado nutricional de seu filho $9,23-29$.

Tabela 4. Razão de Prevalência (RP) ajustada e Intervalo de Confiança de 95\% (IC95\%) da subestimação materna do peso da criança segundo variáveis infantis e maternas (n=673). Goiânia (GO), 2012.

\begin{tabular}{|c|c|c|}
\hline Variáveis & RP ajustada (IC95\%) & $p$ \\
\hline \multicolumn{3}{|l|}{ Idade da criança } \\
\hline$<24$ meses & 1,00 & \\
\hline$\geq 24$ meses & $1,29(1,02-1,64)$ & 0,035 \\
\hline \multicolumn{3}{|l|}{ Prematuridade } \\
\hline $\operatorname{sim}$ & 1,00 & \\
\hline Não & $0,79(0,55-1,13)$ & 0,190 \\
\hline \multicolumn{3}{|l|}{ Primogênito } \\
\hline Sim & 1,00 & \\
\hline Não & $1,11(0,85-1,44)$ & 0,434 \\
\hline \multicolumn{3}{|c|}{ Estado nutricional da criança } \\
\hline Sem excesso de peso & 1,00 & \\
\hline Com excesso de peso & $2,70(2,13-3,41)$ & $<0,001$ \\
\hline \multicolumn{3}{|l|}{ Idade da mãe } \\
\hline$<20$ anos & 1,00 & \\
\hline$\geq 20$ anos & $1,30(0,71-2,37)$ & 0,396 \\
\hline \multicolumn{3}{|l|}{ Escolaridade da mãe } \\
\hline Ensino fundamental & 1,00 & \\
\hline Ensino médio & $0,83(0,63-1,10)$ & 0,202 \\
\hline Ensino superior & $0,63(0,44-0,92)$ & 0,017 \\
\hline \multicolumn{3}{|c|}{ Estado nutricional da mãe } \\
\hline Baixo peso & 1,00 & \\
\hline Eutrófica & $0,77(0,51-1,17)$ & 0,219 \\
\hline Excesso de peso & $0,64(0,41-0,99)$ & 0,048 \\
\hline
\end{tabular}

\section{I S C U S S Ã O}

No presente estudo, foi encontrada uma distorção na percepção materna do peso da criança, sendo que as mães tendem a subestimar o peso das crianças com peso normal ou excesso de peso. Os fatores associados positivamente à subestimação materna do peso da criança foram idade e excesso de peso da criança, enquanto a escolaridade e o peso materno apresentaram associação negativa.

Na comparação dos resultados deste trabalho com estudos que avaliaram a percepção materna do peso de crianças menores de cinco anos de idade, observou-se que, na maioria desses, há uma importante subestimação materna do peso de crianças com excesso de peso ${ }^{9,23-29}$. A mesma observação foi feita pelo presente estudo: dentre as crianças com excesso de peso, 62,5\% foram reconhecidas pelas mães como eutróficas ou baixo peso. Esse resultado também foi semelhante ao encontrado em estudo em escolas da cidade de Vitória (ES) - com crianças de outra faixa etária (entre 7 e 10 anos de idade) -, no qual $65,9 \%$ das crianças com sobrepeso tiveram seu peso subestimado pelas mães ${ }^{5}$, e, em estudo canadense, no qual $63,0 \%$ dos pais de escolares com excesso de peso também subestimaram o peso de seus filhos ${ }^{30}$.

A subestimação materna do peso corporal dos filhos pode ser explicada pela falta de conhecimento sobre o assunto, pela não compreensão dos gráficos e das curvas utilizados pelos profissionais de saúde no diagnóstico nutricional da criança ou mesmo por falta de entendimento do significado do termo excesso de peso $2,7,13,29$. Em geral, os pais parecem mais preocupados com a condição da criança estar abaixo do peso do que com o peso elevado, o que pode resultar em um incentivo ao abuso de comida, principalmente por parte da mãe, que costuma ser a principal cuidadora ${ }^{2}$.

No presente estudo, observou-se também que $66 \%$ das mães classificaram adequadamente 
Tabela 5. Descrição de estudos transversais que analisaram a subestimação materna do peso de crianças menores de cinco anos de idade.

\begin{tabular}{|c|c|c|c|c|}
\hline $\begin{array}{l}\text { Autor (ano) } \\
\text { País }\end{array}$ & Amostra estudada & $\begin{array}{l}\text { Faixa etária } \\
\text { (anos) }\end{array}$ & $\begin{array}{l}\text { \% de subestimação segundo } \\
\text { estado nutricional }\end{array}$ & $\begin{array}{c}\text { Fatores associados à } \\
\text { subestimação do estado } \\
\text { nutricional } \\
\end{array}$ \\
\hline Presente estudo & $\begin{array}{l}\mathrm{n}=673 \\
\text { (crianças da área urbana de uma } \\
\text { capital brasileira) }\end{array}$ & $0-5$ & $\begin{array}{l}\text { 24,8\%: para crianças sem ex- } \\
\text { cesso de peso } \\
62,5 \% \text { : para crianças com ex- } \\
\text { cesso de peso }\end{array}$ & $\begin{array}{l}\text { Idade da Criança (+) } \\
\text { Escore-Z IMC/Idade da criança (+) } \\
\text { IMC materno (-) } \\
\text { Escolaridade materna (-) }\end{array}$ \\
\hline $\begin{array}{l}\text { Carvalhaes \& Godoy } 9 \\
\text { (2002) } \\
\text { Brasil }\end{array}$ & $\begin{array}{l}\mathrm{n}=860 \\
\text { (crianças de UBS da área urba- } \\
\text { na de um município) }\end{array}$ & $0-2$ & $\begin{array}{l}86,4 \% \text { : para crianças com ex- } \\
\text { cesso de peso }\end{array}$ & Nenhum fator associado \\
\hline $\begin{array}{l}\text { Baughcum et al. }{ }^{18} \\
\text { (2006) } \\
\text { Estados Unidos }\end{array}$ & $\begin{array}{l}\mathrm{n}=622 \\
\text { (crianças de clínicas privadas e } \\
\text { do WIC) }\end{array}$ & $2-5$ & $\begin{array}{l}79,0 \% \text { : para crianças com ex- } \\
\text { cesso de peso }\end{array}$ & Escolaridade materna (-) \\
\hline $\begin{array}{l}\text { Chaparro et al. }{ }^{23} \\
\text { (2011) } \\
\text { Estados Unidos }\end{array}$ & $\begin{array}{l}n=1702 \\
\text { (crianças do WIC) }\end{array}$ & $3-5$ & $\begin{array}{l}95,6 \% \text { : para crianças com so- } \\
\text { brepeso }\end{array}$ & $\begin{array}{l}\text { IMC materno }(+) \\
\text { Peso ao nascer }(+)\end{array}$ \\
\hline $\begin{array}{l}\text { Oude et al. }{ }^{24} \text { (2010) } \\
\text { Holanda }\end{array}$ & $\begin{array}{l}\mathrm{n}=439 \\
\text { (crianças de escolas da área ur- } \\
\text { bana e rural) }\end{array}$ & $4-5$ & $\begin{array}{l}78,2 \% \text { : para crianças obesas } \\
75,0 \% \text { : para crianças com } \\
\text { excesso de peso } 50 \% \text { : para } \\
\text { crianças obesas }\end{array}$ & Não investigado \\
\hline $\begin{array}{l}\text { Manios et al. }{ }^{25} \text { (2009) } \\
\text { Grécia }\end{array}$ & $\begin{array}{l}\mathrm{n}=2287 \\
\text { (crianças de creches públicas e } \\
\text { privadas de cinco municípios) }\end{array}$ & $2-5$ & $\begin{array}{l}88,3 \% \text { : para crianças com ris- } \\
\text { co de sobrepeso } \\
54,5 \% \text { : para crianças com so- } \\
\text { brepeso }\end{array}$ & $\begin{array}{l}\text { Escore-Z IMC/I da criança (+) } \\
\text { Sexo masculino (+) } \\
\text { Atividade física da criança (-) } \\
\text { Escolaridade materna (-) }\end{array}$ \\
\hline $\begin{array}{l}\text { Hackie \& } \text { Bowles }^{26} \\
\text { (2007) } \\
\text { Estados Unidos }\end{array}$ & $\begin{array}{l}\mathrm{n}=38 \\
\text { (crianças com sobrepeso/WIC) }\end{array}$ & $2-5$ & $\begin{array}{l}61,5 \% \text { : para crianças com so- } \\
\text { brepeso }\end{array}$ & Nenhum fator associado \\
\hline $\begin{array}{l}\text { Campbell et al..27 } \\
\text { (2006) } \\
\text { Austrália }\end{array}$ & $\begin{array}{l}\mathrm{n}=324 \\
\text { (crianças de coorte de Centro de } \\
\text { Saúde, áreas urbana e semi-ru- } \\
\text { ral) }\end{array}$ & 4 & $\begin{array}{l}71,0 \% \text { : para crianças com so- } \\
\text { brepeso }\end{array}$ & Não investigado \\
\hline $\begin{array}{l}\text { Carnell et al. }{ }^{28} \text { (2005) } \\
\text { Inglaterra }\end{array}$ & $\begin{array}{l}\mathrm{n}=564 \\
\text { (crianças de } 16 \text { escolas primá- } \\
\text { rias) }\end{array}$ & $3-5$ & $\begin{array}{l}98,1 \% \text { : para crianças com so- } \\
\text { brepeso } \\
82,3 \% \text { : para crianças obesas }\end{array}$ & Nenhum fator associado \\
\hline
\end{tabular}

Nota: n: tamanho amostral; (+) associação positiva; (-) associação negativa; WIC: Special Supplemental Nutrition Program for Women, Infants, and Children; IMC/I: Índice de Massa Corporal por Idade; UBS: Unidade Básica de Saúde.

o estado nutricional de seus filhos. Warschburger \& Kröller $^{3}$, em pesquisa realizada com mães de crianças de um a dez anos de idade em clínicas de internação e creches, mostraram que apenas metade das mães identificou corretamente as silhuetas com excesso de peso de seus filhos, bem como os riscos relacionados à saúde; outro estudo realizado no nordeste brasileiro indicou que crianças acima do peso são vistas como possuidoras de boa saúde e de melhores cuidados dos pais ${ }^{11}$.

Para muitas mães, o excesso de peso da criança é sinônimo de saúde, pois acreditam que a criança seja mais saudável quando está acima do peso, o que a tornará mais resistente às doenças. Entretanto, esse é um conceito equivocado que causa tantos prejuízos à saúde da criança quanto o baixo peso ${ }^{31}$.

Em relação aos fatores associados à subestimação do peso da criança pela mãe, observou-se que, com o aumento da idade da criança, a percepção materna tornou-se menos coerente. Esse fato pode ser justificado pela diminuição da frequência com que a criança vai às consultas de acompanhamento de crescimento com o passar da idade, o que faz a mãe perder o referencial do 
peso da criança. Segundo o calendário mínimo de consultas para assistência à criança, proposto pelo Ministério da Saúde, a partir do segundo ano de vida, recomenda-se o mínimo de uma consulta para acompanhamento do crescimento, enquanto, no primeiro ano de vida, o recomendado são sete consultas e, no segundo ano de vida, duas consultas ${ }^{32}$.

No entanto, Tenório \& Cobayashi3 ${ }^{33}$ encontraram resultados contraditórios. Segundo esses autores, pais de crianças menores têm a convicção de que, com o crescimento, o peso se tornará adequado e, portanto, não há motivos para preocupação ou intervenção.

A escolaridade materna apresentou associação positiva com o estado nutricional das crianças e associação negativa com a subestimação materna do peso da criança neste estudo. Em estudo de Pakpour et al. ${ }^{2}$, o nível de escolaridade da mãe e a renda familiar são apontados como os dois principais fatores que determinam a percepção da mãe sobre a obesidade infantil. Segundo esses autores, o nível de escolaridade está correlacionado ao nível de conhecimento preexistente a respeito da obesidade infantil e dos seus riscos relacionados, sendo as mães com mais anos de estudo mais prováveis de considerar o peso dos filhos como um problema. Baughcum et al. ${ }^{29}$, em estudo com um grupo de mães com filhos de 23 a 60 meses de idade e percentil de IMC/I superior a 95, verificaram que, entre as mães com baixo nível de escolaridade, somente $16 \%$ delas acreditavam que os filhos apresentavam excesso de peso.

Em relação ao estado nutricional materno, observou-se no presente estudo que as mães com excesso de peso identificaram mais adequadamente o peso de seus filhos. Chaparro et al. ${ }^{23}$, no entanto, analisando crianças de 36 a 60 meses de idade, observaram que o IMC materno foi negativamente associado à precisão da percepção materna do peso, e os estudos de Carnell et al. ${ }^{28}$ e Baughcum et al. ${ }^{29}$ não encontraram associação entre essa variável e a percepção materna do peso da criança. A divergência de resultados entre os estudos na avaliação da associação entre a obesidade materna e a percepção do peso da criança sugere a necessidade de investigar outros fatores que podem interferir nessa relação, como a percepção que a mãe tem do seu próprio peso.

Embora não tenha havido associação com a subestimação materna neste estudo, o sexo da criança também pode estar relacionado a maior subestimação do peso. Em geral, as mães tendem a subestimar mais o excesso de peso dos meninos do que o das meninas. Normalmente, o excesso de peso dos meninos é associado a uma vantagem física, e isso pode estar relacionado à diferença na composição corporal entre os gêneros. No entanto, parece mais provável ser um reflexo dos valores sociais - em que as mulheres são levadas a se preocuparem mais com a estética corporal do que os homens -, o que aponta para um cuidado maior da mãe sobre o peso das filhas ${ }^{7,13,27,34}$.

Uma limitação do nosso estudo foi não ter utilizado escalas de imagem para a análise da percepção materna do peso da criança. Esse fato é discutido por Rietmeijer-Mentink et al. ${ }^{35}$, que mostraram que estudos que utilizam essa forma de avaliação mostram maior percentual de acerto da percepção dos pais em relação às crianças com sobrepeso em comparação com estudos em que se utilizam descrições verbais.

Isto sugere que os pais têm maior dificuldade em reconhecer o estado nutricional de seu filho com excesso de peso, por não querer rotulá-lo verbalmente com excesso de peso. A razão para essa relutância pode estar relacionada a uma associação negativa com a expressão 'acima do peso' devido ao estigma das pessoas obesas na sociedade. Outra hipótese é a de que as mães podem se sentir desconfortáveis quando respondem a uma questão sobre o peso de seus filhos, devido aos fatores emocionais que podem interferir durante o processo de avaliação ${ }^{35,36}$.

Entretanto, em outro estudo em que também foram utilizados dois instrumentos dife- 
rentes, um verbal e um visual, para avaliação da percepção materna do peso, o instrumento verbal mostrou ser mais preciso do que o visual em relação à correta classificação do peso das crianças pelas mães. Apenas 15,0\% das mães que foram questionadas verbalmente subestimaram o estado nutricional de seus filhos, contra $41,3 \%$ que subestimaram o peso e responderam de acordo com fotografias representativas da imagem corporal das crianças $^{34}$.

Outra limitação do estudo foi a utilização do peso e da altura referidos para a avaliação do estado nutricional materno. Entretanto, estudo de Peixoto et al. ${ }^{21}$ observou alta concordância entre as medidas referidas de peso e estatura em relação às medidas aferidas, concluindo que, em estudos epidemiológicos de monitoramento da prevalência de excesso de peso, o peso e a altura referidos por indivíduos de 20 a 64 anos de idade podem ser consideradas informações confiáveis.

\section{O N C L U S Ã O}

Existe uma distorção acentuada na percepção materna do peso de crianças menores de cinco anos; a subestimação materna do peso da criança apresentou associação direta com idade e estado nutricional da criança e associação inversa com escolaridade e estado nutricional materno. Esse fato é preocupante uma vez que a dificuldade dos pais em reconhecer o excesso de peso dos filhos contribui para o aumento do excesso de peso nas crianças. Sugere-se mais atenção no atendimento a essas mães a fim de orientá-las na identificação do real estado nutricional da criança, evitando assim prejuízos futuros para a saúde de seus filhos.

\section{OLABORADORES}

TPDA FREITAS participou da coleta, processamento, análise e interpretação dos dados, revisão da literatura e redação do manuscrito. LLS SILVA contribuiu na análise e interpretação dos dados e redação da versão final do manuscrito. GS TELES participou da coleta, processamento, análise e interpretação dos dados, revisão da literatura e redação do manuscrito. MRG PEIXOTO contribuiu na concepção do estudo, coordenação da coleta de dados, análise e interpretação dos dados e revisão crítica do manuscrito. IHCF MENEZES contribuiu na concepção do estudo, coordenação da coleta de dados e revisão crítica do manuscrito.

\section{REFERÊ NCIAS}

1. World Health Organization. Set of recomendations on the marketing of foods and non alcoholic beverages to children. Geneva: WHO; 2010.

2. Pakpour AH, Yekaninejad MS, Chen H. Mother's perception of obesity in schoolchildren: A survey and impact of an education intervention. J Pediatr. 2011; 87(2):169-74.

3. Warschburger $P$, Kröller K. Childhood overweight and obesity: Maternal perceptions of the time for engaging in child weight management. BMC Public Health. 2012; 12:295.

4. Daniels SR, Arnett DK, Eckel RH, Gidding SS, Hayman LL, Kumanyika $S$, et al. Overweight in children and adolescents: Pathophysiology, consequences, prevention, and treatment. Circulation. 2005; 111(15):1999-2012.

5. Molina MCB, Faria CP, Montero P, Cade NV. Correspondence between children's nutritional status and mothers' perceptions: A population based study. Cad Saúde Pública. 2009; 25(10): 2285-90.

6. Frota MA, Barroso MGT. Repercussão da desnutrição na família. Rev Latino-Am Enfermagem. 2005; 13(6):996-1000.

7. Maynard LM, Galuska DA, Blanck HM, Serdula MK. Maternal perceptions of weight status of children. Pediatrics. 2003; 111(5):1226-31.

8. Eckstein KC, Mikhail LM, Ariza AJ, Thomson JS, Binns HJ. Parents perception of their child's weight and health. Pediatrics. 2006; 117(3):681-90.

9. Carvalhaes MABL, Godoy I. As mães sabem avaliar adequadamente o peso das crianças? Rev Nutr. 2002; 15(2):155-62. http//:dx.doi.org/10.1590/S14 15-52732002000200004

10. Huang JS, Becerra K, Oda T, Walker E, Xu R, Donohue $\mathrm{M}$, et al. Parental ability to discriminate the weight status of children: Results of a survey. Pediatrics. 2007; 120(1):112-9.

11. Boa-Sorte N, Néri LA, Leite MEQ, Brito SM, Meirelles AR, Luduvice FBS, et al. Percepção materna e autopercepção do estado nutricional de crianças e 
adolescentes de escolas privadas. J Pediatr. 2007; 83(4):349-56

12. Francescatto C, Santos NS, Coutinho VF, Costa RF. Mothers' perceptions about the nutritional status of their overweight children: A systematic review. J Pediatr. 2014; 90(4):332-43.

13. Giacossomi MC, Zanella T, Hofelmann DA. Percepção materna do estado nutricional de crianças de creches de cidade do Sul do Brasil. Rev Nutr. 2011; 24(5):689-702. http//:dx.doi.org/10.1590/S1 415-52732011000500003

14. Instituto Brasileiro de Geografia e Estatística. Características gerais da população: resultados da amostra. Brasília: IBGE; 2000 [acesso 2014 out 18]. Disponível em: http://www.ibge.gov.br/home/ estatistica/populacao/censo2000/populacao/ censo2000_populacao.pdf

15. Villar J, Ismail LC, Victora CG, Ohuma OE, Bertino E, Altamar DG, et al. International standards for newborn weight, length, and head circumference by gestational age and sex: The Newborn CrossSectional Study of the INTERGROWTH-21 $1^{\text {st }}$ Project. Lancet. 2014; 384(9946):857-68.

16. Olsen IE, Growman SA, Lauson ML, Clark RH, Zemil BS. New intrauterine growth curves based on United States DATA. Pediatrics. 2010; 125(2):e214-24.

17. Lohman TG, Roche AF, Martorel R. Anthropometric standardization reference manual. Champaign (IL): Human Kinetics Books; 1988.

18. World Health Organization. Multicentre Growth Reference Study Group. Child growth standards: Length/height-for-age, weight-for-age, weight-forlength, weight-for-height and body mass index-forage: Methods and development. Geneva: WHO; 2006.

19. World Health Organization. Obesity: Preventing and managing the global epidemic. Geneva: WHO; 1997.

20. World Health Organization. Growth reference data for 5-19 years, WHO reference. Washington (DC): WHO; 2007 [cited 2015 Apr 8]. Available from: http://www.who.int/growthref/en/

21. Peixoto MRG, Benicio MHDA, Jardim PCBV. Validade do peso e da altura auto-referidos: o estudo de Goiânia. Rev Saúde Pública. 2006; 40(6):1065-72.

22. Landis JR, Koch GG. The measurement of observer agreement for categorical data. Biometrics. 1977; 33(1):159-74.

23. Chaparro M, Langellier B, Kim L, Whaley S. Predictors of accurate maternal perception of their preschool child's weight status among hispanic WIC participants. Obesity (Silver Spring). 2011; 19(10): 2026-30.
24. Oude LHGM, Stolk RP, Sauer PJ. How do parents of 4- to 5-year-old children perceive the weight of their children? Acta Pediatr. 2010; 99(2):263-7.

25. Manios Y, Kondaki K, Kourlaba G, Vasilopoulou E, Grammatikaki E. Maternal perceptions of their child's weight status: The GENESIS study. Publ Health Nutr. 2009; 12(8):1099-105.

26. Hackie M, Bowles CL. Maternal perception of their overweight children. Public Health Nurs. 2007; 24(6):538-46.

27. Campbell MW, Williams J, Hampton A, Wake M. Mother's concern and preceptions of overweight in Australian preschool-aged children. Med J Aust. 2006; 184(6):274-7.

28. Carnell S, Edwards C, Croker H, Boniface D, Wardle J. Parental perceptions of overweight in 3-5 y olds. Int J Obes. 2005; 29(4):353-5.

29. Baughcum AE, Chamberlin LA, Deeks CM, Powers SW, Withaker RC. Maternal perceptions of overweight preschool children. Pediatrics. 2000; 106(6):1380-6.

30. He M, Evans A. Are parents aware that their children are overweight or obese? Can Fam Physician. 2007; 53(9):1493-9.

31. Chuproski P, Mello DF. Percepção materna do estado nutricional de seus filhos. Rev Nutr. 2009; 22(6):929-93. http://dx.doi.org/10.1590/\$1415-52 732009000600014

32. Brasil. Ministério da Saúde. Secretaria de Políticas de Saúde. Departamento de Atenção Básica. Saúde da criança: acompanhamento do crescimento e desenvolvimento infantil. Brasília: Ministério da Saúde; 2002.

33. Tenório AS, Cobayashi F. Obesidade infantil na percepção dos pais. Rev Paul Pediatr. 2011; 29(4):634-9.

34. Moschonis G, Latridi V, Mavrogianni C, Siatitsa PE, Kyriakou A, Dede V, et al. Accuracy and correlates of visual and verbal instruments assessing maternal perceptions of children's weight status: The Healthy Growth Study. Public Health Nutr. 2011; 14(11):1979-87.

35. Rietmeijer-Mentink M, Paulis W, Middelkoop M, Bindels P, Wouden J. Difference between parental perception and actual weight status of children: A systematic review. Matern Child Nutr. 2013; 9(1):3-22.

36. Lazzeri G, Casorelli A, Giallombar D, Grasso A, Guidoni C, Menoni E, et al. Nutritional surveillance in Tuscany: Maternal perception of nutritional status of 8-9 y-old school-children. J Prev Med Hyg. 2006; 47(1):16-21. 
